\title{
How Complex Is the Concanavalin A-Carboxypeptidase Y Interaction?
}

\author{
Katarzyna Herman, ${ }^{\dagger}$ Marek Weiss, ${ }^{\dagger}$ Małgorzata Lekka, ${ }^{\dagger}$ and Arkadiusz Ptak $^{\dagger *}$ \\ ${ }^{\dagger}$ Institute of Physics, Faculty of Technical Physics, Poznan University of Technology, Piotrowo 3, PL-60965 \\ Poznan, Poland \\ ${ }^{ \pm}$Department of Biophysical Microstructures, Institute of Nuclear Physics, Polish Academy of Sciences, PL- \\ 31342 Cracow, Poland \\ "Corresponding Author: arkadiusz.ptak@put.poznan.pl
}

\section{Supporting Information}

\section{Carboxypeptidase Y (CPY)}

CPY from baker's yeast (Saccharomyces cerevisiae) contains four oligosaccharide chains linked to a single polypeptide chain (Fig. S1). ${ }^{1,2}$ CPY is distinguishable from other carboxypeptidases by the absence of metal ions. The carbohydrate moiety of CPY constitutes about $15 \%$ of the protein molecular mass and is composed of mannose residues, whose presence allows binding to ConA. ${ }^{3}$ As most of peptidases, CPY is highly resistant to changes in the environmental conditions like $\mathrm{pH}$ or temperature. ${ }^{4}$

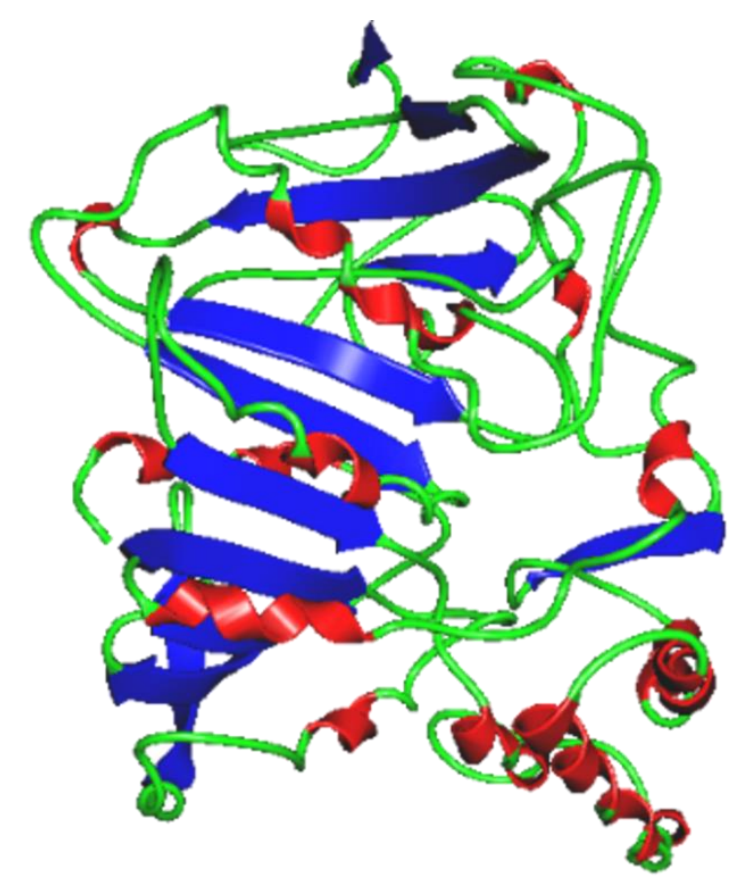

Figure S1. Amino-acid structure of carboxypeptidase Y (CPY;PDB ID: 1YSC)

The binding sites of oligosaccharide chains correspond to the asparagine residues: Asn-13, Asn87, Asn-168 and Asn-368. Two N-acetylglucosamines and 14 up to 18 mannose molecules are linked together to Asn-13, Asn-168 and Asn-368, with the exception of asparagine 87, to which 9 to 11 mannose molecules are attached. Unlike the chain molecules, which are attached to three remaining 
asparagine residues ( 2 molecules per chain are phosphorylated), mannose combined with Asn-87 is not phosphorylated. The total mass of the oligosaccharide chains located in CPY structure is about 10 $\mathrm{kDa}, 20 \%$ of which represents mannose attached to the Asn-87.,6 The CPY structure contains about $38 \%$ of helices (19 helices, 162 amino-acid residues) and 15\% of beta-sheets (19 strands, 67 aminoacid residues). ${ }^{7}$

\section{Concanavalin A (ConA)}

ConA is a member of the legume lectin family. The presence of $\mathrm{Mn}^{2+}$ and $\mathrm{Ca}^{2+}$ ions stabilizes the conformation of the protein due to the strong $\mathrm{pH}$ susceptibility of ConA. In acidic environment, at pH 6 and below, ConA exists as a dimer, whereas in alkaline environment, when $\mathrm{pH}$ exceeds 7.5 , as a tetramer or higher aggregates. ${ }^{8,9}$ The tetrameric structure of ConA consists of four identical monomers $(26.5 \mathrm{kDa})$, each of which consists of 237 amino acids and has one saccharide and two metal binding sites (Fig. S2). The protein may therefore bind to mannose as well as interact with immunoglobulins, lipoproteins or rhodopsin.

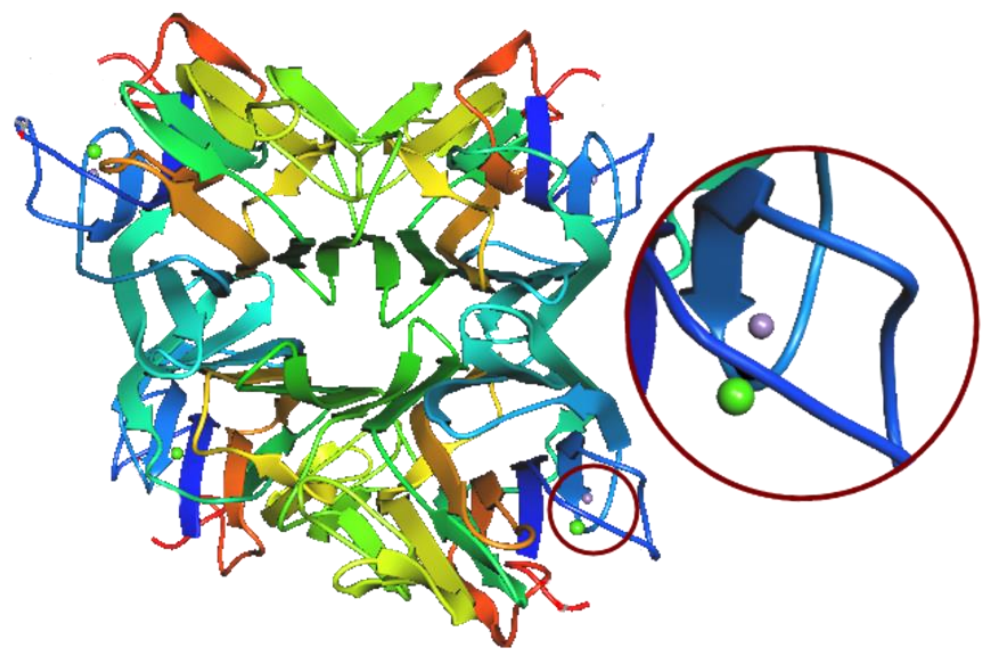

Figure S2. The tetrameric structure of concanavalin A (ConA; PDB ID: 3CNA) and metal ions: $\mathrm{Mn}^{2+}$ (purple ball) and $\mathrm{Ca}^{2+}$ (green ball) in the active center of the protein

The metals are necessary for saccharide-binding activity. ${ }^{10}$ Each metal is surrounded by four amino-acid residues and two water molecules. $\mathrm{Mn}^{2+}$ binds to Glu-8, Asp-10, Asp-19 and His-24. $\mathrm{Ca}^{2+}$ is linked to Asp-10, Tyr-12, Asn-14 and Asp-19. ${ }^{11}$ The saccharide binding ability can be affected by removing metal ions from the lectin's structure. The demetallization of ConA leads to conformational changes in the metal ion binding site. Some of the residues are moved $17 \AA$ in respect to its native position. Such high mobility leads saccharides to bind to ConA on the position of previously removed metal ion. ${ }^{12}$ The protein has the following secondary structure: $5 \%$ of the polypeptide chains are helices (4 helices, 13 amino-acid residues) and 45\% are in form of beta-sheet (18 strands, 109 aminoacid residues). ${ }^{13}$ 


\section{Specific ConA-CPY interaction}

Lectins interact with carbohydrates through hydrogen and coordination metal bonds as well as by Van der Waals and hydrophobic interactions. ConA possess conserved Asp-Asn-Arg triad which is essential in the process of carbohydrates binding (Fig. S3). The Arg-228 residue forms hydrogen bonds with oxygens $\mathrm{O} 3$ and $\mathrm{O} 4$. Moreover, $\mathrm{O} 6$ of the mannose ligand forms a hydrogen bond with Tyr-100 in ConA. The presence of an aromatic ring provides additional stabilization of the complex. ${ }^{14}$

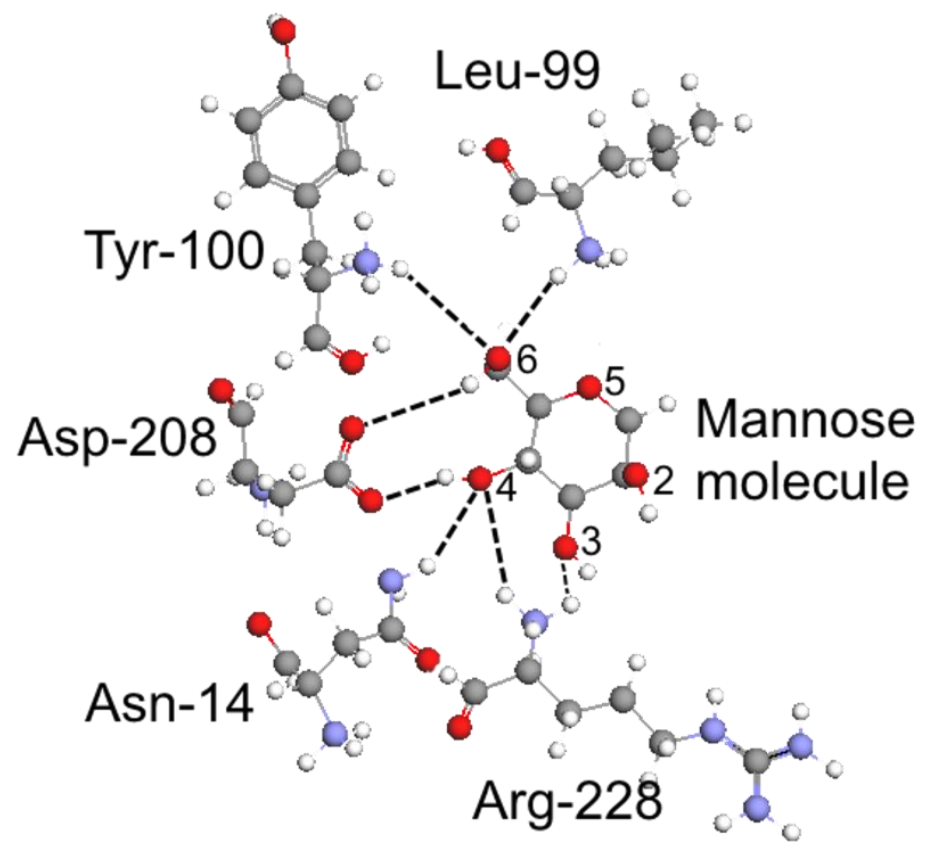

Figure S3. Scheme of the specific binding site between D-mannose and ConA residues (hydrogen bonds are marked with dashed lines)

\section{Reference measurement}

In order to confirm the presence of the specific interactions, a reference measurement in $5 \mathrm{mM}$ ethylenediaminetetraacetic acid (EDTA; Novazym Polska s.c., Poland) solution was performed. The main task of the solution was to mask the metal ions and thereby reduce the ConA conformational stability. The total number of force curves in the reference measurement was 8192 , the same as in the standard measurement (in TBS buffer, $\mathrm{pH}$ 7.6). All these measurements were carried out in force spectroscopy mode at $1 \mu \mathrm{m} / \mathrm{s}$ separation rate. The number of force curves indicating single specific interactions decreased from $30 \%$ in the standard measurement to $8 \%$ in the reference measurement (Fig. S4). Moreover, the number of force curves without any detectable adhesion events grew to $70 \%$ from $5 \%$ in the standard measurement (Table S1). 


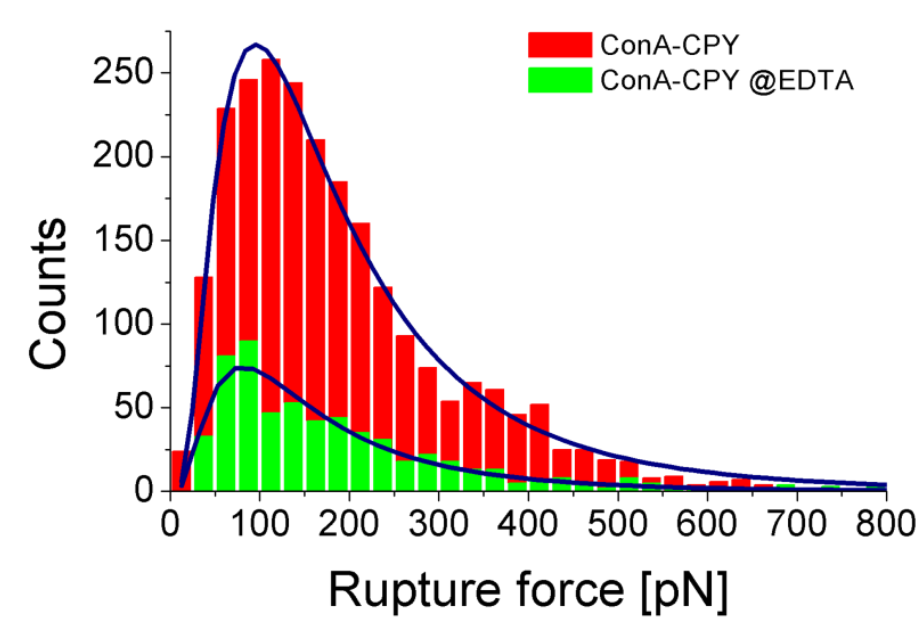

Figure S4. Comparison of histograms obtained for the standard ConA-CPY measurement in TBS buffer (red) and the reference measurement in EDTA solution (green)

Table S1. Results of the reference measurement in relation to the total number of analyzed force curves

\begin{tabular}{|l|c|c|}
\hline Force curves: & ConA-CPY@TBS & ConA-CPY@EDTA \\
\hline single specific interactions & $30 \%$ & $<8 \%$ \\
\hline $\begin{array}{l}\text { multi-specific and non-specific interactions } \\
\text { (rejected from further analysis) }\end{array}$ & $65 \%$ & $22 \%$ \\
\hline $\begin{array}{l}\text { no detectable interactions } \\
\text { (rejected from further analysis) }\end{array}$ & $<5 \%$ & $70 \%$ \\
\hline
\end{tabular}

\section{Acknowledgments}

Visualizations of proteins (Figures S1-S3) have been made with ArgusLab 4.0 (Mark A. Thompson, Planaria Software LLC, Seattle, WA).

\section{References}

(1) Dumoulin, M.; Ueno, H.; Hayashi, R.; Balny, C. Contribution of the Carbohydrate Moiety to Conformational Stability of the Carboxypeptidase Y: High Pressure Study. Eur. J. Biochem. 1999, 262 (2), 475-483.

(2) Hashimoto, C.; Cohen, R. E.; Zhang, W. J.; Ballou, C. E. Carbohydrate Chains on Yeast Carboxypeptidase Y Are Phosphorylated. Proc Natl Acad Sci U S A 1981, 78 (4), 2244-8.

(3) Shiba, Y.; Ichikawa, K.; Serizawa, N.; Yoshikawa, H. Extracellular Processing of Carboxypeptidase Y Secreted by a Saccharomyces Cerevisiae Ssl1 Mutant Strain. J. Ferment. Bioeng. 1998, 86 (6), 545-549. 
(4) Shimizu, H.; Ueno, H.; Hayashi, R. Role of Carbohydrate Moiety in Carboxypeptidase Y: Structural Study of Mutant Enzyme Lacking Carbohydrate Moiety. Biosci. Biotechnol. Biochem. 1999, 63 (6), 1045-1050.

(5) Ballou, L.; Hernandez, L. M.; Alvarado, E.; Ballou, C. E. Revision of the Oligosaccharide Structures of Yeast Carboxypeptidase Y. Proc Natl Acad Sci U S A 1990, 87 (9), 3368-72.

(6) Winther, J. R.; Stevens, T. H.; Kielland-Brandt, M. C. Yeast Carboxypeptidase Y Requires Glycosylation for Efficient Intracellular Transport, but Not for Vacuolar Sorting, in Vivo Stability, or Activity. Eur. J. Biochem. 1991, 197 (3), 681-9.

(7) Endrizzi, J. A.; Breddam, K.; Remington, S. J. 2.8-Å Structure of Yeast Serine Carboxypeptidase. Biochemistry 1994, 33 (37), 11106-20.

(8) McKenzie, G. H.; Sawyer, W. H. The Binding Properties of Dimeric and Tetrameric Concanavalin A. J. Biol. Chem. 1973, 248 (2), 549-556.

(9) Pflumm, M. N.; Beychok, S. Alkali and Urea Induced Conformation Changes in Concanavalin A. Biochemistry 1974, 13, 4982-4987.

(10) Kalb, A.; Levitzki, A. Metal-Binding Sites of Concanavalin A and Their Role in the Binding of a-Methyl d-Glucopyranoside. Biochem. J. 1968, 109 (4), 669-72.

(11) Reeke, G. N.; Becker, J. W.; Cunningham, B. A.; Gunther, G. R.; Wang, J. L.; Edelman, G. M. Relationships Between The Structure And Activities Of Concanavalin A. Ann. N. Y. Acad. Sci. 1974, 234 (0), 369-82.

(12) Kaushik, S.; Mohanty, D.; Surolia, A. The Role of Metal Ions in Substrate Recognition and Stability of Concanavalin A: A Molecular Dynamics Study. Biophys. J. 2009, 96 (1), 21-34.

(13) Naismith, J. H.; Field, R. A. Structural Basis of Trimannoside Recognition by Concanavalin A. J. Biol. Chem. 1996, 271 (2), 972-976.

(14) Loris, R.; Hamelryck, T.; Bouckaert, J.; Wyns, L. Legume Lectin Structure. Biochimica et Biophysica Acta - Protein Structure and Molecular Enzymology. 1998, 1383 (1), 9-36. 